\title{
On Generalized Moduli of Quasi-Banach Space
}

\section{Young Chel Kwun $\mathbb{D}^{1},{ }^{1}$ Hussain Minhaj Uddin Ahmad Qadri, ${ }^{2}$ Waqas Nazeer $(\mathbb{D})^{3}$ Absar Ul Haq, ${ }^{4}$ and Shin Min Kang $\mathbb{D D}^{5,6}$}

\author{
${ }^{1}$ Department of Mathematics, Dong-A University, Busan 49315, Republic of Korea \\ ${ }^{2}$ Aitchison College, Lahore 54000, Pakistan \\ ${ }^{3}$ Division of Science and Technology, University of Education, Lahore 54000, Pakistan \\ ${ }^{4}$ Department of Mathematics, University of Management and Technology, Sialkot Campus, Sialkot 51410, Pakistan \\ ${ }^{5}$ Department of Mathematics and RINS, Gyeongsang National University, Jinju 52828, Republic of Korea \\ ${ }^{6}$ Center for General Education, China Medical University, Taichung 40402, Taiwan
}

Correspondence should be addressed to Waqas Nazeer; nazeer.waqas@ue.edu.pk and Shin Min Kang; smkang@gnu.ac.kr

Received 23 November 2017; Accepted 7 March 2018; Published 3 May 2018

Academic Editor: P. Veeramani

Copyright (C) 2018 Young Chel Kwun et al. This is an open access article distributed under the Creative Commons Attribution License, which permits unrestricted use, distribution, and reproduction in any medium, provided the original work is properly cited.

We shall discuss three generalized moduli such as generalized modulus of convexity, modulus of smoothness, and modulus of ZouCui of quasi-Banach spaces and give some important properties of these moduli. Furthermore, we establish relationships of these generalized moduli with each other.

\section{Introduction}

The study on Banach space geometry provides many fundamental notions and interesting aspects and sometimes has surprising results. The basic geometric properties such as convexity, smoothness, and nonsquareness have made great contributions to various fields of Banach space theory. Strict convexity of Banach spaces was first introduced in 1936 by Clarkson [1] (and independently by Akhiezer and Krein) as the property that the unit sphere contains no nontrivial line segments; that is, $1-\left\|2^{-1}(x+y)\right\|>0$ whenever $\|x\|=\|y\|=1$. Clarkson [1] made use of these values to define the "uniform" version of convexity to look at how "convex" the unit ball is in a space. And the modulus of convexity provides a quantification of the geometric structure of the space from the viewpoint of convexity. A situation similar to this also occurs in smoothness and other properties. A Banach space $X$ is said to be smooth if each unit vector has a unique norm one support functional. In fact, this is equivalent to the statement that the norm is Gateaux differentiable. This allows us to quantify the geometric structure of the space from the viewpoint of smoothness, namely, the modulus of smoothness of a Banach space $X$. An advantage of these quantifications is that the complete duality between uniform convexity and uniform smoothness can be easily deduced by the well-known Lindenstrauss formulas; that is, a Banach space $X$ is uniformly convex if and only if its dual space $X^{*}$ is uniformly smooth. The same statement still holds if $X$ is replaced with $X^{*}$. Thus quantifying geometric structures might lead to better results. Note that the same duality does not hold between strict convexity and smoothness in general, though one of those two properties of $X^{*}$ implies the other of $X$. There are some other ideas to quantify geometric structures of Banach spaces.

In [2], the authors claim that modulus of convexity and generalized convexity mold have dual relationship, and generalized convexity mold has many excellent properties.

In [3], the authors study a generalized modulus of convexity where certain related geometrical properties of this modulus are analyzed in Banach spaces.

In $[4,5]$, the modulus of Yang-Wang was introduced in Banach spaces.

In [6], the modulus of Zuo-Cui was introduced in Banach spaces. The author proved many results with this special type of modulus. 
The most recent research work at this topic can be consulted from $[7,8]$.

\section{Preliminaries}

There are lots of quantitative descriptions of geometrical properties of quasi-Banach spaces. The most common way for creating these descriptions is to define a real function (a modulus) and a suitable coefficient or constant closely related to this function, depending on the space structure under consideration. Some of the moduli and their related coefficients (or characteristics) for quasi-Banach spaces have also been investigated so far. These moduli are the attempts in order to get a better understanding of the two facts about the space:

(i) The shape of the unit ball of the concerned space.

(ii) The conditions and relations for convergence of sequences.

The most recent research work with these moduli is investigated by $[7,8]$.

Definition 1. For a quasi-Banach space $\mathscr{B}$, the modulus of convexity is a function $\delta_{\mathscr{B}}:(0,2] \rightarrow[0,1]$ defined as

$$
\begin{aligned}
& \delta_{\mathscr{B}}(\epsilon)=\inf \left\{1-\frac{\left\|x_{1}+x_{2}\right\|}{2 C}: \epsilon \in[0,2], C \geq 1, x_{1}, x_{2}\right. \\
& \left.\in S_{\mathscr{B}}, \frac{\left\|x_{1}-x_{2}\right\|}{C} \geq \epsilon\right\} .
\end{aligned}
$$

A characteristic or related coefficient of this modulus is

$$
\delta_{0}(\mathscr{B})=\sup \left\{\epsilon \in[0,2]: \delta_{\mathscr{B}}(\epsilon)=0\right\} .
$$

Definition 2. Let $\alpha \in(0,1)$ and $\epsilon \in[0,2]$. For a quasi-Banach space $\mathscr{B}$, the generalized modulus of convexity is a function $\delta_{\mathscr{B}}^{(\alpha)}:(0,2] \rightarrow[0,1]$ defined as

$$
\begin{gathered}
\delta_{\mathscr{B}}^{(\alpha)}(\epsilon)=\inf \left\{1-\frac{\left\|(\alpha) x_{1}+(1-\alpha) x_{2}\right\|}{C}: C\right. \\
\left.\geq 1, x_{1}, x_{2} \in S_{\mathscr{B}}, \frac{\left\|x_{1}-x_{2}\right\|}{C} \geq \epsilon\right\} .
\end{gathered}
$$

A characteristic or related coefficient of this modulus is

$$
\delta_{\theta}(\mathscr{B})=\sup \left\{\epsilon \in[0,2]: \delta_{\mathscr{B}}^{(\alpha)}(\epsilon)=0\right\} .
$$

Definition 3. For a quasi-Banach space $\mathscr{B}$, the modulus of smoothness is a function $\rho_{\mathscr{B}}:[0, \infty) \rightarrow[0, \infty)$ defined as

$$
\begin{aligned}
& \rho_{\mathscr{B}}(t)=\sup \left\{1-\frac{\left\|x_{1}+x_{2}\right\|}{2 C}: x_{1}, x_{2} \in S_{\mathscr{B}}, C\right. \\
& \left.\geq 1, \frac{\left\|x_{1}-x_{2}\right\|}{C} \leq t, t \geq 0\right\} .
\end{aligned}
$$

A characteristic or related coefficient of this modulus is

$$
\begin{aligned}
\rho_{0}(\mathscr{B}) & =\lim _{t \rightarrow 0^{+}}\left(\frac{\rho_{\mathscr{B}}(t)}{t}\right)=\lim _{t \rightarrow 0^{+}}\left(\frac{\rho_{1 \mathscr{B}}(t)}{t}\right) \\
& =\lim _{t \rightarrow 0^{+}}\left(\frac{\rho_{2 \mathscr{B}}(t)}{t}\right) .
\end{aligned}
$$

Definition 4. Let $\alpha \in(0,1)$ and $t \in[0,2]$. For a quasi-Banach space $\mathscr{B}$, the generalized modulus of smoothness is a function $\rho_{\mathscr{B}}^{(\alpha)}:[0, \infty) \rightarrow[0, \infty)$ defined as

$$
\begin{aligned}
& \rho_{\mathscr{B}}^{(\alpha)}(t)=\sup \left\{1-\frac{\left\|(\alpha) x_{1}+(1-\alpha) x_{2}\right\|}{C}: C \geq 1, x_{1}, x_{2}\right. \\
& \left.\quad \in S_{\mathscr{B}}, \frac{\left\|x_{1}-x_{2}\right\|}{C} \leq t\right\}, \\
& \rho_{\mathscr{B}}^{(\alpha)}(t) \\
& \quad=\sup \left\{\frac{\left\|(\alpha) x_{1}-(t / 2) x_{2}\right\|+\left\|(1-\alpha) x_{1}-(t / 2) x_{2}\right\|}{C}\right. \\
& \left.-\frac{1}{C}\right\},
\end{aligned}
$$

where $x_{1}, x_{2} \in S_{\mathscr{B}},\left\|x_{1}\right\| \leq 1,\left\|x_{2}\right\| \leq 1$, and $C \geq 1$.

A characteristic or related coefficient of this modulus is

$$
\rho_{\theta}(\mathscr{B})=\lim _{t \rightarrow 0^{+}}\left(\frac{\rho_{\mathscr{B}}^{(\alpha)}(t)}{t}\right)=\lim _{t \rightarrow 0^{+}}\left(\frac{\rho_{1 \mathscr{B}}^{(\alpha)}(t)}{t}\right) .
$$

Definition 5. Let $t \in[0, \infty)$ and $p \in[1, \infty)$. For a quasiBanach space $\mathscr{B}$, the modulus of $Z u o-C u i$ is a function $\xi_{\mathscr{B}}^{(p)}$ : $(0, \infty) \rightarrow(0, \infty)$ defined as

$$
\begin{aligned}
& \xi_{\mathscr{B}}^{(p)}(t) \\
& \quad=\sup \left\{\left[\frac{\left\|x_{1}+t x_{2}\right\|^{p}+\left\|x_{1}-t x_{2}\right\|^{p}}{2 C^{p}}\right]^{1 / p}, x_{1}, x_{2}\right. \\
& \left.\quad \in S_{\mathscr{B}}, C \geq 1\right\} .
\end{aligned}
$$

A characteristic or related coefficient of this modulus is

$$
\xi_{\theta}(\mathscr{B})=\lim _{t \rightarrow 0}\left(\frac{\xi_{\mathscr{B}}^{(p)}(t)-1 / C}{t}\right) .
$$

\section{Relations Concerning Generalized Modulus of Convexity}

Lemma 6 (see [9]). Every convex function $f$ with convex domain in $\mathbb{R}$ is continuous.

Proposition 7 (see [10]). Let $\mathscr{B}$ be a uniformly convex space. Then for every $d>0, \varepsilon>0$, and for arbitrary vectors, $x_{1}, x_{2} \in$ 
$\mathscr{B}$ with $\left\|x_{1}\right\| \leq d,\left\|x_{2}\right\| \leq d$, and $\left\|x_{1}-x_{2}\right\| / C \geq \varepsilon$, where $C \geq 1$, there exists $\delta>0$ such that

$$
\frac{\left\|x_{1}+x_{2}\right\|}{2 C} \leq\left[1-\delta\left(\frac{\varepsilon}{d}\right)\right] d .
$$

Proposition 8 (see [10]). A nontrivial quasi-Banach space $\mathscr{B}$ is uniformly nonsquare if and only if $\delta_{\mathscr{B}}(\epsilon)>0$.

Proposition 9. Let $\delta_{\mathscr{B}}(\epsilon)$ be the modulus of convexity of a quasi-Banach space $\mathscr{B}$; then

$$
2 \alpha \delta_{\mathscr{B}}(\epsilon) \leq \delta_{\mathscr{B}}^{(\alpha)}(\epsilon) \leq 2(1-\alpha) \delta_{\mathscr{B}}(\epsilon) .
$$

Proof. Let $x, y \in \mathscr{B},\|y\|=C$, and $\|x-y\| / C \geq \epsilon$. Then we have

$$
\begin{aligned}
1-\frac{\|\alpha x+(1-\alpha y)\|}{C} & =1-\frac{\alpha\|x+y\|+(1-2 \alpha)\|y\|}{C} \\
& =1-\frac{\alpha\|x+y\|}{C}-\frac{(1-2 \alpha)\|y\|}{C} \\
& =1-\frac{2 \alpha\|x+y\|}{2 C}-(1-2 \alpha) \\
& \leq 2 \alpha \sup \left\{1-\frac{\|x+y\|}{2 C}\right\} \\
& =2 \alpha\left[\delta_{\mathscr{B}}(\epsilon)\right],
\end{aligned}
$$

and we have obtained the first inequality; now to prove the second one, for any $\eta>0$, there exist $x, y \in S_{\mathscr{B}}$ with $\|x\|=1$, $\|y\|=C$, and $\|x-y\| / C \geq \epsilon$ such that $\|x+y\| / 2 C \leq \delta(\epsilon)+\eta$; then we have

$$
\begin{aligned}
1- & \frac{\|\alpha x+(1-\alpha y)\|}{C}=1-\frac{\alpha\|x+y\|+(1-2 \alpha)\|y\|}{C} \\
& \leq 1-\frac{(1-\alpha)\|x+y\|+(1-2 \alpha)\|y\|-C}{C} \\
& \leq 1-\frac{2(1-\alpha)\|x+y\|}{2 C}+\frac{(1-2 \alpha)\|y\|-C}{C} \\
& \leq 1-\frac{2(1-\alpha)\|x+y\|}{2 C}+2(1-\alpha)-1 \\
& \leq 2(1-\alpha)-\frac{2(1-\alpha)\|x+y\|}{2 C} \\
& \leq 2(1-\alpha)\left[1-\frac{\|x+y\|}{2 C}\right] \leq 2(1-\alpha)\left[\delta_{\mathscr{B}}(\epsilon)\right] .
\end{aligned}
$$

This completes the proof.

Corollary 10. Let $\epsilon_{\theta}$ be the characteristic of generalized modulus of convexity $\delta_{\mathscr{B}}^{(\alpha)}(\epsilon)$ of a quasi-Banach space $\mathscr{B}$. Then

$$
\epsilon_{\theta}(\mathscr{B})=\sup \left\{\epsilon \in(0,2]: \delta_{\mathscr{B}}^{(\alpha)}(\epsilon)=0\right\} .
$$

Theorem 11. A quasi-Banach space $\mathscr{B}$ is uniformly nonsquare if and only if

$$
\delta_{\mathscr{B}}^{(\alpha)}(\epsilon)>0
$$

Proof. By Proposition $8, \mathscr{B}$ is uniformly nonsquare if and only if $\delta_{\mathscr{B}}(\epsilon)>0$, where $\epsilon \in(0,2)$, and by Proposition 9, $\delta_{\mathscr{B}}(\epsilon)>0$ if and only if $\delta_{\mathscr{R}}^{(\alpha)}>0(\epsilon)>0$.

Combining both of these results we get the proof.

Lemma 12. Let $\mathscr{B}$ be a quasi-Banach space and $\epsilon \in(0,2]$. Then the following statements hold:

(1) $\delta_{\mathscr{B}}^{(\alpha)}(\epsilon)$ is convex and continuous function.

(2) $\delta_{\mathscr{B}}^{(\alpha)}(\epsilon)$ is a nondecreasing function.

(3) $\delta_{\mathscr{B}}^{(\alpha)}(\epsilon) / \epsilon$ is a nondecreasing function.

Proof. (1) Let

$$
\epsilon_{2}=2\left(\frac{\epsilon_{2}-\epsilon_{1}}{2-\epsilon_{1}}\right)+\epsilon_{1}\left(1-\frac{\epsilon_{2}-\epsilon_{1}}{2-\epsilon_{1}}\right) .
$$

Then

$$
\begin{aligned}
\delta_{\mathscr{B}}^{(\alpha)}\left(\epsilon_{2}\right)= & \delta_{\mathscr{B}}^{(\alpha)}\left[2\left(\frac{\epsilon_{2}-\epsilon_{1}}{2-\epsilon_{1}}\right)+\epsilon_{1}\left(1-\frac{\epsilon_{2}-\epsilon_{1}}{2-\epsilon_{1}}\right)\right] \\
\leq & \delta_{\mathscr{B}}^{(\alpha)}(2)\left(\frac{\epsilon_{2}-\epsilon_{1}}{2-\epsilon_{1}}\right) \\
& +\delta_{\mathscr{B}}^{(\alpha)}\left(\epsilon_{1}\right)\left(1-\frac{\epsilon_{2}-\epsilon_{1}}{2-\epsilon_{1}}\right) \\
\leq & \delta_{\mathscr{B}}^{(\alpha)}(2)\left(\frac{\epsilon_{2}-\epsilon_{1}}{2-\epsilon_{1}}\right)+\delta_{\mathscr{B}}^{(\alpha)}\left(\epsilon_{1}\right) \\
& -\delta_{\mathscr{B}}^{(\alpha)}\left(\epsilon_{1}\right)\left(\frac{\epsilon_{2}-\epsilon_{1}}{2-\epsilon_{1}}\right) \\
= & \left(\frac{\epsilon_{2}-\epsilon_{1}}{2-\epsilon_{1}}\right)\left[\delta_{\mathscr{B}}^{(\alpha)}(2)-\delta_{\mathscr{B}}^{(\alpha)}\left(\epsilon_{1}\right)\right] \\
& +\delta_{\mathscr{B}}^{(\alpha)}\left(\epsilon_{1}\right) .
\end{aligned}
$$

Now

$$
\begin{aligned}
\delta_{\mathscr{B}}^{(\alpha)}\left(\epsilon_{2}\right)-\delta_{\mathscr{B}}^{(\alpha)}\left(\epsilon_{1}\right) & \leq\left(\frac{\epsilon_{2}-\epsilon_{1}}{2-\epsilon_{1}}\right)\left[1-\delta_{\mathscr{B}}^{(\alpha)}\left(\epsilon_{1}\right)\right] \\
& \leq\left(\frac{\epsilon_{2}-\epsilon_{1}}{2-\epsilon_{1}}\right)[2(1-\alpha)],
\end{aligned}
$$

by using Proposition 9. Hence we get

$$
\delta_{\mathscr{B}}^{(\alpha)}\left(\epsilon_{2}\right)-\delta_{\mathscr{B}}^{(\alpha)}\left(\epsilon_{1}\right) \leq[2(1-\alpha)]\left(\frac{\epsilon_{2}-\epsilon_{1}}{2-\epsilon_{1}}\right) .
$$

Since $\delta_{\mathscr{B}}^{(\alpha)}(\epsilon)$ is convex, so it is continuous by Lemma 6 .

(2) Let $0<\epsilon_{1} \leq \epsilon_{2}<0$ and $x, y \in S_{\mathscr{B}}$ with $\|x-y\| \geq \epsilon_{2}$. Let us consider

$$
\begin{aligned}
& z=\frac{(\alpha x+(1-\alpha) y)}{C}, \\
& t \leq \frac{\epsilon_{1}}{\epsilon_{2}} \\
& u=t x+(1+t) z, \\
& v=t y+(1-t) z .
\end{aligned}
$$


Then $u, v \in S_{\mathscr{B}}$ with $\|u-v\| \geq \epsilon_{1}$; we have

$$
\begin{aligned}
1-\frac{\|\alpha u+(1-\alpha) v\|}{C} & \leq t\left[1-\frac{\|\alpha x+(1-\alpha) y\|}{C}\right] \\
& =\frac{\epsilon_{1}}{\epsilon_{2}}\left[1-\frac{\|\alpha x+(1-\alpha) y\|}{C}\right] ;
\end{aligned}
$$

thus

$$
\begin{aligned}
& \sup \left\{1-\frac{\|\alpha u+(1-\alpha) v\|}{C}\right\} \\
& \leq \frac{\epsilon_{1}}{\epsilon_{2}}\left[\sup \left\{1-\frac{\|\alpha x+(1-\alpha) y\|}{C}\right\}\right],
\end{aligned}
$$

which implies that

$$
\delta_{\mathscr{B}}^{(\alpha)}\left(\epsilon_{1}\right) \leq \frac{\epsilon_{1}}{\epsilon_{2}}\left[\delta_{\mathscr{B}}^{(\alpha)}\left(\epsilon_{2}\right)\right] \quad\left(\frac{\delta_{\mathscr{B}}^{(\alpha)}\left(\epsilon_{1}\right)}{\epsilon_{1}} \leq \frac{\delta_{\mathscr{B}}^{(\alpha)}\left(\epsilon_{2}\right)}{\epsilon_{2}}\right)
$$

(3) Let $0 \leq t_{1} \leq t_{2} \leq 2$; we have

$$
t_{1} \delta_{\mathscr{B}}^{(\alpha)}\left(t_{2}\right) \leq t_{2} \delta_{\mathscr{B}}^{(\alpha)}\left(t_{1}\right) \leq t_{1} \delta_{\mathscr{B}}^{(\alpha)}\left(t_{1}\right)
$$

Since $\delta_{\mathscr{B}}^{(\alpha)}\left(t_{1}\right)>0$, so we have

$$
\delta_{\mathscr{B}}^{(\alpha)}\left(t_{2}\right)<\delta_{\mathscr{B}}^{(\alpha)}\left(t_{1}\right)
$$

This completes the proof.

Theorem 13. Let $\mathscr{B}$ be a uniformly convex space and $\alpha \epsilon$ $(0,1)$. Then for every $d>0, \varepsilon>0$ and for arbitrary vectors, $x_{1}, x_{2} \in \mathscr{B}$ with $\left\|x_{1}\right\| \leq d,\left\|x_{2}\right\| \leq d$, and $\left\|x_{1}-x_{2}\right\| / C \geq \varepsilon$, there exists $\delta=(\varepsilon / d)>0$ such that

$$
\begin{aligned}
& \frac{\left\|(\alpha) x_{1}+(1-\alpha) x_{2}\right\|}{C} \\
& \quad \leq\left[1-2\left(1-C+C \delta\left(\frac{\epsilon}{d}\right) \cdot \min \{\alpha, 1-\alpha\}\right)\right] \frac{d}{C} .
\end{aligned}
$$

Proof. Without loss of generality, assume that $\alpha \in(0,1 / 2]$ so

$$
\begin{aligned}
\frac{\left\|(\alpha) x_{1}+(1-\alpha) x_{2}\right\|}{C}= & \frac{\alpha\left(x_{1}+x_{2}\right)+(1-2 \alpha)\left\|x_{2}\right\|}{C} \\
= & 2 \alpha \frac{\left\|x_{1}+x_{2}\right\|}{2 C} \\
& +\frac{(1-2 \alpha)\left\|x_{2}\right\|}{C} .
\end{aligned}
$$

Now by Proposition 7, there exist $\delta>0$ such that, for $\|y\| \leq d$,

$$
\frac{\left\|x_{1}+x_{2}\right\|}{2 C} \leq\left[1-\delta\left(\frac{\epsilon}{d}\right)\right] d .
$$

Therefore, we get

$$
\begin{aligned}
& \frac{\left\|(\alpha) x_{1}+(1-\alpha) x_{2}\right\|}{C} \\
& \leq 2 \alpha\left(1-\delta\left(\frac{\epsilon}{d}\right)\right) d+\frac{(1-2 \alpha) d}{C} \\
& =\left[2 \alpha\left(1-\delta\left(\frac{\epsilon}{d}\right)\right)+\frac{(1-2 \alpha)}{C}\right] d \\
& =\left[2 \alpha-2 \alpha \delta\left(\frac{\epsilon}{d}\right)+\frac{(1-2 \alpha)}{C}\right] d \\
& =\left[\frac{(1-2 \alpha+2 \alpha C)}{C}-2 \alpha \delta\left(\frac{\epsilon}{d}\right)\right] d \\
& =\frac{1}{C}-\left[\frac{2 \alpha}{C}+\frac{2 \alpha C}{C}-2 \alpha \delta\left(\frac{\epsilon}{d}\right)\right] d \\
& =\frac{1}{C}-\frac{2}{C}\left[1-C+C \delta\left(\frac{\epsilon}{d}\right) \cdot \alpha\right] d \\
& =\left[1-2\left(1-C+C \delta\left(\frac{\epsilon}{d}\right) \cdot \min \{\alpha, 1-\alpha\}\right)\right] \frac{d}{C} .
\end{aligned}
$$

This completes the proof.

\section{Relations Concerning Generalized Modulus of Smoothness}

Theorem 14. Let $\mathscr{B}$ be a quasi-Banach space. Then for every $x \in \mathscr{B},\|x\|=1$, and $x^{*} \in X^{*},\left\|x^{*}\right\|=1$

$$
\begin{aligned}
& \rho_{\mathscr{B}}^{(\alpha)}(t)=\sup \left\{\frac{t \epsilon}{2}-\delta_{\mathscr{B}^{*}}^{(\alpha)}(\epsilon): t>0, \epsilon \in(0,2]\right\}, \\
& \rho_{\mathscr{B}^{*}}^{(\alpha)}(t)=\sup \left\{\frac{t \epsilon}{2}-\delta_{\mathscr{B}}^{(\alpha)}(\epsilon): t>0, \epsilon \in(0,2]\right\} .
\end{aligned}
$$

Proof. Throughout the proof of the first part, we take

$$
\begin{aligned}
x, y & \in S_{\mathscr{B}}, \\
\|x\| & \leq 1, \\
\|y\| & \leq 1, \\
\alpha & \in(0,1), \\
t & \geq 0, \\
C & \geq 1, \\
\left\|f^{*}\right\| & \leq 1, \\
\left\|g^{*}\right\| & \leq 1,
\end{aligned}
$$

$$
f, g \in S_{\mathscr{B}^{*}}
$$

$\left\|x^{*}\right\| \leq 1$

$\left\|y^{*}\right\| \leq 1$

$$
x^{*}, y^{*} \in S_{\mathscr{B}^{*}} .
$$


Take

$$
\begin{aligned}
\rho_{\mathscr{B}}^{(\alpha)}(t)=\sup \left\{\left\|\left(\frac{\alpha}{C}\right) x+\left(\frac{t}{2 C}\right) y\right\|\right. \\
\left.+\left\|\left(\frac{1-\alpha}{C}\right) x-\left(\frac{t}{2 C}\right) y\right\|-1\right\}=\sup \left\{f \left[\left(\frac{\alpha}{C}\right) x\right.\right. \\
\left.\left.+\left(\frac{t}{2 C}\right) y\right]+g\left[\left(\frac{1-\alpha}{C}\right) x-\left(\frac{t}{2 C}\right) y\right]-1\right\} \\
=\frac{1}{C} \sup \left\{f\left[(\alpha x)+\left(\frac{t}{2}\right) y\right]\right. \\
\left.+g\left[(1-\alpha) x-\left(\frac{t}{2}\right) y\right]-C\right\}=\frac{1}{C} \\
+\sup \left\{\left[(\alpha f)+\left(\frac{t}{2}\right) g\right] x+\left[\frac{t}{2}(f-g)\right] y-C\right\} \\
=\frac{1}{C} \sup \left\{\|(\alpha) f+(1-\alpha) g\|+\frac{t}{2}\|f-g\|-C\right\} \\
=\sup \left\{\frac{\|(\alpha) f+(1-\alpha) g\|}{C}+\frac{t}{2 C}\|f-g\|-1\right\} \\
=\sup \left\{\frac{t}{2 C}\|f-g\|-\left[1-\frac{\|(\alpha) f+(1-\alpha) g\|}{C}\right]\right\} \\
=\sup \left\{\frac{t \epsilon C}{2 C}-\left[\delta_{\mathscr{B}^{*}}^{(\alpha)}(\epsilon)\right]\right\}=\sup \left\{\frac{t \epsilon}{2}-\left[\delta_{\mathscr{B}^{*}}^{(\alpha)}(\epsilon)\right]\right\} .
\end{aligned}
$$

Hence, we get

$$
\rho_{\mathscr{B}}^{(\alpha)}(t)=\sup \left\{\frac{t \epsilon}{2}-\delta_{\mathscr{B}^{*}}^{(\alpha)}(\epsilon): t>0, \epsilon \in(0,2]\right\} .
$$

This completes the first part of the proof.

To prove the second part, let us start as

$$
\begin{aligned}
& \rho_{\mathscr{B}^{*}}^{(\alpha)}(t)=\sup \left\{\left\|\left(\frac{\alpha}{C}\right) f+\left(\frac{t}{2 C}\right) g\right\|\right. \\
& \left.+\left\|\left(\frac{1-\alpha}{C}\right) g-\left(\frac{t}{2 C}\right) g\right\|-1\right\} \\
& =\sup \left\{\left[\left(\frac{\alpha f}{C}\right)+\left(\frac{t g}{2 C}\right)\right] x\right. \\
& \left.+\left[\left(\frac{(1-\alpha) f}{C}\right)-\left(\frac{t g}{2 C}\right)\right] y-1\right\}=\frac{1}{C} \\
& \cdot \sup \left\{f[(\alpha x)+(1-\alpha) y]+g\left[\frac{t}{2}(x-y)\right]-C\right\} \\
& =\frac{1}{C} \sup \left\{\left[(\alpha f)+\left(\frac{t}{2}\right) g\right] x+\frac{t}{2}[g(x-y)]-C\right\} \\
& =\frac{1}{C} \sup \left\{\|(\alpha) x+(1-\alpha) y\|+\frac{t}{2}\|x-y\|-C\right\} \\
& =\sup \left\{\frac{\|(\alpha) x+(1-\alpha) y\|}{C}+\frac{t}{2 C}\|x-y\|-1\right\}
\end{aligned}
$$

$$
\begin{aligned}
& =\sup \left\{\frac{t}{2 C}\|x-y\|-\left[1-\frac{\|(\alpha) x+(1-\alpha) y\|}{C}\right]\right\} \\
& =\sup \left\{\frac{t \epsilon C}{2 C}-\left[\delta_{\mathscr{B}}^{(\alpha)}(\epsilon)\right]\right\}=\sup \left\{\frac{t \epsilon}{2}-\left[\delta_{\mathscr{B}}^{(\alpha)}(\epsilon)\right]\right\} .
\end{aligned}
$$

Lemma 15. Let $\mathscr{B}$ be a quasi-Banach space and $\tau \in(0,2]$. Then the following statements hold:

(1) $\rho_{\mathscr{B}}^{(\alpha)}(\tau)$ is convex and continuous function.

(2) $\rho_{\mathscr{B}}^{(\alpha)}(\tau)$ is a nondecreasing function.

(3) $\rho_{\mathscr{B}}^{(\alpha)}(\tau) / \tau$ is a nondecreasing function.

Proof. (1) Since $\rho_{\mathscr{B}}^{(\alpha)}(\tau)$ is convex, so it is continuous by Lemma 6.

(2) Let $x_{1}, x_{2} \in S_{\mathscr{B}}, 0<\tau_{1}<\tau_{2}$, and $\alpha \in(0,1)$.

Now let

$$
\begin{aligned}
x_{1}= & \frac{\alpha\left(\tau_{2}-\tau_{1}\right)}{\tau_{2}+\alpha\left(\tau_{1}-\tau_{2}\right)}\left[(1-\alpha) x+\left(\frac{\tau_{1}}{2 C}\right) y\right], \\
x_{2}= & (1-\alpha) x+\left(\frac{\tau_{1}}{2 C}\right) y, \\
x_{3}= & {\left[\frac{\alpha(1-\alpha)\left(\tau_{2}-\tau_{1}\right)}{\tau_{2}+\alpha\left(\tau_{1}-\tau_{2}\right)}\right] x } \\
& +\left[\frac{\tau_{2}\left(\tau_{2}-\tau_{1}\right)(1-\alpha)}{2\left[\tau_{2}+\alpha\left(\tau_{1}-\tau_{2}\right)\right]}\right] y, \\
x_{4}= & (1-\alpha) x+\left(\frac{\tau_{2}}{2 C}\right) y, \\
x_{5}= & \alpha x+\left(\frac{\tau_{1}}{2 C}\right) y, \\
x_{6}= & {\left[\frac{\alpha \tau_{1}}{\tau_{2}+\alpha\left(\tau_{1}-\tau_{2}\right)}\right] x } \\
& +\left[\frac{\tau_{1} \tau_{2}}{2\left[\tau_{2}+\alpha\left(\tau_{1}-\tau_{2}\right)\right]}\right] y, \\
x_{7}= & \alpha x+\left(\frac{\tau_{2}}{2 C}\right) y .
\end{aligned}
$$

Now

$$
\begin{aligned}
x_{3}+x_{4} & =x_{1}+x_{2}, \\
\left\|x_{3}\right\|+\left\|x_{4}\right\| & \geq\left\|x_{1}+x_{2}\right\|, \\
x_{5}-x_{1} & =x_{6}, \\
\left\|x_{5}\right\|-\left\|x_{1}\right\| & \geq\left\|x_{6}\right\| .
\end{aligned}
$$

From (38) and (40), we get

$$
\left\|x_{3}\right\|+\left\|x_{4}\right\|+\left\|x_{6}\right\| \leq\left\|x_{5}\right\|+\left\|x_{2}\right\| .
$$


Similarly,

$$
\begin{gathered}
x_{3}-x_{6}=x_{7}, \\
\left\|x_{3}\right\|-\left\|x_{6}\right\| \geq\left\|x_{7}\right\| .
\end{gathered}
$$

Therefore, we have

$$
\begin{aligned}
& \left\|x_{5}\right\|+\left\|x_{2}\right\| \leq\left\|x_{4}\right\|-\left\|x_{7}\right\|, \\
& \alpha x+\left(\frac{\tau_{1}}{2 C}\right) y+(1-\alpha) x+\left(\frac{\tau_{1}}{2 C}\right) y \\
& \quad \leq(1-\alpha) x+\left(\frac{\tau_{2}}{2 C}\right) y+\alpha x+\left(\frac{\tau_{2}}{2 C}\right) y,
\end{aligned}
$$

which implies that

$$
\begin{aligned}
& \left\|\alpha x+\left(\frac{\tau_{1}}{2 C}\right) y\right\|+\left\|(1-\alpha) x-\left(\frac{\tau_{1}}{2 C}\right) y\right\| \\
& \quad \leq\left\|\alpha x+\left(\frac{\tau_{2}}{2 C}\right) y\right\|+\left\|(1-\alpha) x-\left(\frac{\tau_{2}}{2 C}\right) y\right\| ;
\end{aligned}
$$

thus we have

$$
\rho_{\mathscr{B}}^{(\alpha)}\left(\tau_{1}\right) \leq \rho_{\mathscr{B}}^{(\alpha)}\left(\tau_{2}\right) .
$$

This shows that $\rho_{\mathscr{B}}^{(\alpha)}(\tau)$ is a nondecreasing function.

(3) This part is an immediate consequence of Theorem 14.

Theorem 16. For a nontrivial quasi-Banach space $\mathscr{B}$ with $t \in$ $[0, \infty)$ and $\alpha \in(0,1)$, one has

$$
\max \{0, t+|2 \alpha-1|-1\} \leq \rho_{\mathscr{B}}^{(\alpha)}<t .
$$

Proof. Let $x, y \in S_{\mathscr{B}}$ and $\alpha \in(0,1)$. Then, we have

$$
\begin{aligned}
& \rho_{\mathscr{B}}^{(\alpha)}(t)=\sup \left\{\left\|(\alpha) x+\left(\frac{t}{2 C}\right) y\right\|\right. \\
& \left.+\left\|(1-\alpha) x-\left(\frac{t}{2 C}\right) y\right\|-\frac{1}{C}\right\} \leq(\alpha)\|x\|+\left(\frac{t}{2 C}\right) \\
& \cdot\|y\|+(1-\alpha)\|x\|-\left(\frac{t}{2 C}\right)\|y\|-\frac{1}{C} \leq\|x\|+t \\
& \quad \leq t .
\end{aligned}
$$

This proves the first inequality; now to prove the second inequality, we proceed as

$$
\begin{aligned}
& \left\|(\alpha) x+\left(\frac{t}{2 C}\right) y-(1-\alpha) x+\left(\frac{t}{2 C}\right) y\right\|-\frac{1}{C} \\
& =(2 \alpha-1)+t-\frac{1}{C} .
\end{aligned}
$$

Also

$$
\begin{aligned}
& \left\|(\alpha) x+\left(\frac{t}{2 C}\right) y-(1-\alpha) x+\left(\frac{t}{2 C}\right) y\right\|-\frac{1}{C} \\
& \leq\left\|(\alpha) x+\left(\frac{t}{2 C}\right) y\right\|+\left\|(1-\alpha) x-\left(\frac{t}{2 C}\right) y\right\| \\
& \quad-\frac{1}{C} .
\end{aligned}
$$

Therefore,

$$
\begin{aligned}
(2 \alpha-1)+t-\frac{1}{C} \leq & \left\|(\alpha) x+\left(\frac{t}{2 C}\right) y\right\| \\
& +\left\|(1-\alpha) x-\left(\frac{t}{2 C}\right) y\right\|-\frac{1}{C} \\
& \leq \rho_{\mathscr{B}}^{(\alpha)}(t) .
\end{aligned}
$$

This completes the second inequality. Now, combining both of the above inequalities and taking the max of the latter, we get

$$
\max \{0, t+|2 \alpha-1|-1\} \leq \rho_{\mathscr{B}}^{(\alpha)}<t
$$

This completes the proof.

Theorem 17. A quasi-Banach space $\mathscr{B}$ is uniformly smooth if and only if

$$
\rho_{\theta}(\mathscr{B})=\lim _{t \rightarrow 0^{+}}\left(\frac{\rho_{\mathscr{B}}^{(\alpha)}(t)}{t}\right)=0 .
$$

Proof. One has

$$
\begin{aligned}
\alpha x & +2(1-\alpha) \frac{t}{2} y=\left[\frac{1}{2} x+\frac{t}{2} y\right]+\alpha x+2(1-\alpha) \frac{t}{2} y \\
& -\left[\frac{1}{2} x+\frac{t}{2} y\right]\left\|\alpha x+2(1-\alpha) \frac{t}{2} y\right\|+\|(1-\alpha) x \\
& +2(1-\alpha) \frac{t}{2} y\|\leq\| \frac{1}{2} x+\frac{t}{2} y\|+\| \alpha x \\
& +2(1-\alpha) \frac{t}{2} y\|-\| \frac{1}{2} x+\frac{t}{2} y\|+\|(1-\alpha) x \\
& +2(1-\alpha) \frac{t}{2} y\|\leq\| \frac{1}{2} x+\frac{t}{2} y\|+\|\left(\alpha-\frac{1}{2}\right) x \\
& +(1-2 \alpha) \frac{t}{2} y\|+\|(1-\alpha) x+2(1-\alpha) \frac{t}{2} y \| \\
& \leq\left\|\frac{1}{2} x+\frac{t}{2} y\right\|+\|\left(\alpha-\frac{1}{2}\right) x \\
& +(1-2 \alpha) \frac{t}{2} y(1-\alpha) x+2(1-\alpha) \frac{t}{2} y\|\leq\| \frac{1}{2} x \\
& +\frac{t}{2} y\|+\| \frac{1}{2} x-\frac{t}{2} y \| .
\end{aligned}
$$

Hence we get

$$
\begin{aligned}
& \left\|\alpha x+2(1-\alpha) \frac{t}{2} y\right\|+\left\|(1-\alpha) x+2(1-\alpha) \frac{t}{2} y\right\| \\
& \leq\left\|\frac{1}{2} x+\frac{t}{2} y\right\|+\left\|\frac{1}{2} x-\frac{t}{2} y\right\| .
\end{aligned}
$$

Dividing by " $C$ " and taking supremum on both sides, we get 


$$
\begin{aligned}
& \sup \left\{\frac{\|\alpha x+2(1-\alpha)(t / 2) y\|+\|(1-\alpha) x+2(1-\alpha)(t / 2) y\|-1}{C}\right\} \\
& \quad \leq \sup \left\{\frac{\|(1 / 2) x+(t / 2) y\|+\|(1 / 2) x-(t / 2) y\|-1}{C}\right\} ;
\end{aligned}
$$

thus

$$
\begin{aligned}
& \sup \left\{\frac{\|\alpha x+2(1-\alpha)(t / 2) y\|+\|(1-\alpha) x+2(1-\alpha)(t / 2) y\|}{C}\right. \\
& \left.-\frac{1}{C}\right\} \leq \sup \left\{\frac{\|x+t y\|+\|x-t y\|}{2 C}-\frac{1}{C}\right\},
\end{aligned}
$$

which implies that

$$
\rho_{\mathscr{B}}^{(\alpha)}[2(1-\alpha) t] \leq \rho_{\mathscr{B}}(t) .
$$

Now

$$
\begin{aligned}
\frac{1}{2} x- & {\left[\frac{1}{2 \alpha}\right] \frac{t}{2} y } \\
= & {\left[\frac{t}{2} y-(1-\alpha x)\right] } \\
& +\left[(1-\alpha x)-\frac{t}{2} y\right]\left\|\frac{1}{2} x+\left[\frac{1}{2 \alpha}\right] \frac{t}{2} y\right\| \\
& +\left\|\frac{1}{2} x-\left[\frac{1}{2 \alpha}\right] \frac{t}{2} y\right\| \\
= & \left\|\frac{1}{2} x+\left[\frac{1}{2 \alpha}\right] \frac{t}{2} y\right\|+\left\|\left[\frac{t}{2} y-(1-\alpha x)\right]\right\| \\
& +\left\|\left[(1-\alpha x)-\frac{t}{2} y\right]\right\| .
\end{aligned}
$$

This can be expressed as

$$
\begin{aligned}
& \frac{\|x+[1 / 2 \alpha] t y\|+\|x-[1 / 2 \alpha] t y\|}{2} \\
& =\left\|\frac{1}{2} x+\left[\frac{1}{2 \alpha}\right] \frac{t}{2} y\right\|+\left\|\left[\frac{t}{2} y-(1-\alpha x)\right]\right\| \\
& +\left\|\left[(1-\alpha x)-\frac{t}{2} y\right]\right\| \\
& \leq\left\|\frac{1}{2} x+\left[\frac{1}{2 \alpha}\right] \frac{t}{2} y\right\| \\
& +\left\|\left[\frac{t}{2} y-(1-\alpha x)\right]+\left[(1-\alpha x)-\frac{t}{2} y\right]\right\| \\
& =\left\|\alpha x+\left[\frac{t}{2}\right] y\right\|+\left\|(1-\alpha) x+\left[\frac{t}{2}\right] y\right\| .
\end{aligned}
$$

Dividing by " $C$ " and taking supremum on both sides

$$
\begin{array}{r}
\sup \left\{\frac{\|x+[1 / 2 \alpha] t y\|+\|x-[1 / 2 \alpha] t y\|-1}{2 C}\right\} \\
=\frac{\|\alpha x+[t / 2] y\|+\|(1-\alpha) x+[t / 2] y\|-1}{C},
\end{array}
$$

thus

$$
\rho_{\mathscr{B}}\left(\frac{1}{2 \alpha} t\right) \leq \rho_{\mathscr{B}}^{(\alpha)}(t) .
$$

Therefore, from (57) and (61) we get

$$
\lim _{t \rightarrow 0^{+}}\left(\frac{\rho_{\mathscr{B}}(t)}{t}\right)=0=\lim _{t \rightarrow 0^{+}}\left(\frac{\rho_{\mathscr{B}}^{(\alpha)}(t)}{t}\right) .
$$

This completes the proof.

\subsection{Relations Concerning Modulus of Zuo-Cui}

Theorem 18. Let $\mathscr{B}$ be a nontrivial quasi-Banach space and $p \in[1, \infty)$. Then

$$
\xi_{\mathscr{B}}^{(p)}(t) \geq \rho_{\mathscr{B}}(t)+\frac{1}{C} .
$$

Proof. By using convexity of the function $f(u)=u^{p}$ on $(0, \infty)$, one can easily obtain

$$
\begin{aligned}
& \left(\frac{\left\|x_{1}+t x_{2}\right\|+\left\|x_{1}-t x_{2}\right\|}{2}\right)^{p} \\
& \leq \frac{\left\|x_{1}+t x_{2}\right\|^{p}+\left\|x_{1}-t x_{2}\right\|^{p}}{2},
\end{aligned}
$$

so,

$$
\begin{aligned}
& \left(\frac{\left\|x_{1}+t x_{2}\right\|+\left\|x_{1}-t x_{2}\right\|}{2 C}\right)^{p} \\
& \leq \frac{\left\|x_{1}+t x_{2}\right\|^{p}+\left\|x_{1}-t x_{2}\right\|^{p}}{2 C^{p}}
\end{aligned}
$$

which implies that

$$
\frac{1}{C}+\rho_{\mathscr{B}}(t) \leq \xi_{\mathscr{B}}^{(p)}(t)
$$

For $p=1$, we have $\xi_{\mathscr{B}^{1}}(t)=1 / C+\rho_{\mathscr{B}}(t)$.

For $p=2$, we have $2\left[C \xi_{\mathscr{B}}^{(2)}(t)\right]^{2}=E(t, \mathscr{B})$, where

$E(t, \mathscr{B})$

$$
=\sup \left\{\left(\left\|x_{1}+t x_{2}\right\|^{2}+\left\|x_{1}-t x_{2}\right\|^{2}\right): x_{1}, x_{2} \in S_{\mathscr{B}}\right\} .
$$


Proposition 19. For a Banach space $\mathscr{B}$,

$$
\xi_{\mathscr{B}}(t)
$$

$$
\begin{aligned}
& =\sup \left\{\left[\frac{\left\|x_{1}+t x_{2}\right\|^{p}+\left\|x_{1}-t x_{2}\right\|^{p}}{2 C}\right]^{1 / p}: x_{1}, x_{2}\right. \\
& \left.\in S_{\mathscr{B}}, t \in[0,1], C \geq 1\right\} .
\end{aligned}
$$

Proof. Consider a convex function defined by

$$
f(t)=\frac{\left\|x_{1}+t x_{2}\right\|^{p}+\left\|x_{1}-t x_{2}\right\|^{p}}{2 C} .
$$

Let $0<t_{1} \leq t_{2}<1$ and $x_{1}, x_{2} \in S_{\mathscr{B}}$. Then

$$
\begin{aligned}
\xi_{\mathscr{B}}\left(t_{1}\right) & =\left\|x_{1}+t_{1} x_{2}\right\|^{p}+\left\|x_{1}-t_{1} x_{2}\right\|^{p}=2 C f\left(t_{1}\right) \\
& =2 C f\left(\frac{t_{2}+t_{1}}{2 t_{2}}\left(t_{2}\right)+\frac{t_{2}-t_{1}}{2 t_{2}}\left(-t_{2}\right)\right) \\
& \leq\left\|x_{1}+t_{2} x_{2}\right\|^{p}+\left\|x_{1}-t_{2} x_{2}\right\|^{p}=2 C f\left(t_{2}\right) \\
& =\xi_{\mathscr{B}}\left(t_{2}\right) .
\end{aligned}
$$

This shows that $\xi_{\mathscr{B}}\left(t_{1}\right) \leq \xi_{\mathscr{B}}\left(t_{2}\right)$. Therefore, we have

$$
\begin{aligned}
& \frac{1}{p} \sup _{x_{1} \in S_{\mathscr{B}}} \sup _{x_{2} \in B_{\mathscr{B}}}\left\{\frac{\left\|x_{1}+t x_{2}\right\|^{p}+\left\|x_{1}-t x_{2}\right\|^{p}}{2 C}\right\} \\
& \quad \leq \xi_{\mathscr{B}}\left(t\left\|x_{2}\right\|\right) \leq \xi_{\mathscr{B}}(t) .
\end{aligned}
$$

Since the opposite inequality holds obviously, to get the first inequality let

$$
g(\lambda)=\frac{\left\|\lambda x_{1}+t x_{2}\right\|^{p}+\left\|\lambda x_{1}-t x_{2}\right\|^{p}}{2 C} .
$$

Since $g(\lambda)$ is a convex and an even function, therefore $g(\lambda) \geq$ $g(1)$. Now for $x_{1}, x_{2} \in B_{\mathscr{B}}$ we have

$$
\begin{aligned}
& \left\|\frac{x_{1}}{\left\|x_{1}\right\|}+t x_{2}\right\|^{p}+\left\|\frac{x_{1}}{\left\|x_{1}\right\|}-t x_{2}\right\|^{p} \\
& \geq\left\|x_{1}+t x_{2}\right\|^{p}+\left\|x_{1}-t x_{2}\right\|^{p} \\
& \geq \sup _{x_{1} \in S_{\mathscr{B}}} \sup _{x_{2} \in B_{\mathscr{B}}}\left\{\left\|x_{1}+t x_{2}\right\|^{p}+\left\|x_{1}-t x_{2}\right\|^{p}\right\} \\
& \geq \sup _{x_{1}, x_{2} \in B_{\mathscr{B}}}\left\{\left\|x_{1}+t x_{2}\right\|^{p}+\left\|x_{1}-t x_{2}\right\|^{p}\right\} \\
& \geq \sup _{x_{1}, x_{2} \in B_{\mathscr{B}}}\left\{\frac{\left\|x_{1}+t x_{2}\right\|^{p}+\left\|x_{1}-t x_{2}\right\|^{p}}{2 C}\right\},
\end{aligned}
$$

which obtains the second inequality. This completes the proof.

Lemma 20. For any quasi-Banach space $\mathscr{B}$ and any $1 \leq p<$ $\infty$, then the following statements hold:
(1) $\xi_{\mathscr{B}}^{(p)}(t)$ is a nondecreasing function.

(2) $\xi_{\mathscr{B}}^{(p)}(t)$ is convex and continuous function.

(3) $\left(\xi_{\mathscr{B}}^{(p)}(t)-1\right) / t$ is a nondecreasing function.

Proof. (1) Let $g(t)=\left\|x_{1}+t x_{2}\right\|^{p}+\left\|x_{1}-t x_{2}\right\|^{p}$ be a convex and even function. Let $0<t_{1} \leq t_{2}$ and $x_{1}, x_{2} \in S_{\mathscr{B}}$. Then we have

$$
\begin{aligned}
\| x_{1} & +t_{1} x_{2}\left\|^{p}+\right\| x_{1}-t_{1} x_{2} \|^{p}=g\left(t_{1}\right) \\
& =g\left(\frac{t_{2}+t_{1}}{2 t_{2}} t_{2}+\frac{t_{2}-t_{1}}{2 t_{2}}\left(-t_{2}\right)\right) \\
& \leq \frac{t_{2}+t_{1}}{2 t_{2}} g\left(t_{2}\right)+\frac{t_{2}-t_{1}}{2 t_{2}} g\left(t_{2}\right)=g\left(t_{2}\right) \\
& =\left\|x_{1}+t_{2} x_{2}\right\|^{p}+\left\|x_{1}-t_{2} x_{2}\right\|^{p} \leq 2 C^{p} \xi_{\mathscr{B}}^{(p)}\left(t_{2}\right),
\end{aligned}
$$

which implies that

$$
\frac{\left\|x_{1}+t_{1} x_{2}\right\|^{p}+\left\|x_{1}-t_{1} x_{2}\right\|^{p}}{2 C^{p}} \leq \xi_{\mathscr{B}}^{(p)}\left(t_{2}\right) .
$$

Hence $\xi_{\mathscr{B}}^{(p)}\left(t_{1}\right) \leq \xi_{\mathscr{B}}^{(p)}\left(t_{2}\right)$. Since $\xi_{\mathscr{B}}^{(p)}(t)$ is convex, so it is continuous by Lemma 6 .

(2) Let $x_{1}, x_{2} \in S_{\mathscr{B}}, t_{1}, t_{2}>0$, and $\lambda \in(0,1)$ and $r(s)=$ $\operatorname{sgn}[\sin (2 \pi s)]$. Then we have

$$
\begin{aligned}
\xi_{\mathscr{B}}^{(p)} & {\left[\lambda t_{1}+(1-\lambda) t_{2}\right]=\frac{1}{2 C^{p}}\left[\int_{0}^{1} \| x\right.} \\
& \left.+r(s)\left[\lambda t_{1}+(1-\lambda) t_{2}\right] y \|^{p} d t\right]^{1 / p} \\
& \leq \frac{1}{2 C^{p}}\left[\int _ { 0 } ^ { 1 } \left[\lambda\left\|x+r(s) t_{1} y\right\|\right.\right. \\
& \left.\left.+(1-\lambda)\left\|x+r(s) t_{2} y\right\|\right]^{p} d t\right]^{1 / p} \\
& \leq \frac{\lambda}{2 C^{p}}\left[\int_{0}^{1}\left[\left\|x+r(s) t_{1} y\right\|\right]^{p} d t_{1}\right]^{1 / p} \\
& +\frac{(1-\lambda)}{2 C^{p}}\left[\int_{0}^{1}\left\|x+r(s) t_{2} y\right\|^{p} d t_{2}\right]^{1 / p} \leq \lambda \xi_{\mathscr{B}}^{(p)}\left(t_{1}\right) \\
& +(1-\lambda) \xi_{\mathscr{B}}^{(p)}\left(t_{2}\right) .
\end{aligned}
$$

This shows that $\xi_{\mathscr{B}}^{(p)}(t)$ is convex.

(3) Let $0<t_{1} \leq t_{2}<0$ and $\lambda \in(0,1)$ with $t_{1}=\lambda t_{2}$. Then

$$
\frac{\xi_{\mathscr{B}}^{(p)}\left(t_{1}\right)-1}{t_{1}} \leq \frac{\xi_{\mathscr{B}}^{(p)}\left(\lambda t_{2}\right)-1}{\lambda t_{2}} \leq \frac{\xi_{\mathscr{B}}^{(p)}\left(t_{2}\right)-1}{t_{2}}
$$

This shows that $\left(\xi_{\mathscr{B}}^{(p)}(t)-1\right) / t$ is nondecreasing. This completes the proof.

Proposition 21. Let $\mathscr{B}$ be a quasi-Banach space, $p \in[1, \infty)$, and $t>0$. Then the following conditions are equivalent: 
(1) $\xi_{\mathscr{B}}^{(p)}(t)<1+t$

(2) $\xi\left(t, x_{1}\right)<1+t$.

Proof. (1) $\Rightarrow$ (2) Suppose on the contrary that $\xi\left(t, x_{1}\right) \geq 1+t$; it is enough to take $\left(t, x_{1}\right)=1+t$. Since

$$
\begin{aligned}
& J(\mathscr{B})=\sup \left\{\frac{1}{C}\right. \\
& \left.\cdot \min \left(\left\|x_{1}+x_{2}\right\|,\left\|x_{1}-x_{2}\right\|\right): x_{1}, x_{2} \in S_{\mathscr{B}}\right\},
\end{aligned}
$$

by using the definition of sup, for any $\epsilon>0$, there exist $x_{1}, x_{2} \in S_{\mathscr{B}}$ such that

$$
\begin{aligned}
& \frac{\left\|x_{1}+t x_{2}\right\|+\left\|x_{1}-t x_{2}\right\|}{2} \\
& \quad \geq \min \left\{\left\|x_{1}+t x_{2}\right\|,\left\|x_{1}-t x_{2}\right\|\right\} \geq c(1+t-\epsilon) .
\end{aligned}
$$

Applying convexity of the function $f(u)=u^{p}$, we get

$$
\begin{aligned}
& {\left[\left(\frac{\left\|x_{1}+t x_{2}\right\|+\left\|x_{1}-t x_{2}\right\|}{2}\right)^{p}\right]^{1 / p}} \\
& \quad \leq\left(\frac{\left\|x_{1}+t x_{2}\right\|^{p}+\left\|x_{1}-t x_{2}\right\|^{p}}{2}\right)^{1 / p} ;
\end{aligned}
$$

therefore from (79)

$$
\begin{aligned}
& \left(\frac{\left\|x_{1}+t x_{2}\right\|^{p}+\left\|x_{1}-t x_{2}\right\|^{p}}{2}\right)^{1 / p} \\
& \quad \geq \min \left\{\left\|x_{1}+t x_{2}\right\|,\left\|x_{1}-t x_{2}\right\|\right\} \geq c(1+t-\epsilon) .
\end{aligned}
$$

Since $\epsilon$ is any arbitrary so

$$
\xi_{\mathscr{B}}^{(p)}(t) \geq 1+t
$$

which leads a contradiction.

$(2) \Rightarrow(1)$ Suppose on the contrary that $\xi_{\mathscr{B}}^{(p)}(t) \geq 1+t$; it is enough to take $\xi_{\mathscr{B}}^{(p)}(t)=1+t$. Again using the definition of sup, for all $\epsilon>0$, there exist $x_{1}, x_{2} \in S_{\mathscr{B}}$ such that

$$
\left\|x_{1}+t x_{2}\right\|^{p}+\left\|x_{1}-t x_{2}\right\|^{p} \geq 2 C^{p}(1+t-\epsilon)^{p},
$$

also using

$$
\left\|x_{1}+t x_{2}\right\|^{p}+\left\|x_{1}-t x_{2}\right\|^{p} \leq 2 C^{p}(1+t)^{p},
$$

thus

$$
\begin{aligned}
2 C^{p}(1+t)^{p} & \geq\left\|x_{1}+t x_{2}\right\|^{p}+\left\|x_{1}-t x_{2}\right\|^{p} \\
& \geq 2 C^{p}(1+t-\epsilon)^{p}
\end{aligned}
$$

since $\epsilon$ is arbitrary, so

$$
\left\|x_{1}+t x_{2}\right\|^{p}+\left\|x_{1}-t x_{2}\right\|^{p}=2 C^{p}(1+t)^{p}
$$

which implies that

$$
\left\|x_{1}+t x_{2}\right\|=\left\|x_{1}-t x_{2}\right\|=C(1+t) \text {. }
$$

So using the definition of $\xi\left(t, x_{1}\right)$ we get $\xi\left(t, x_{1}\right) \geq 1+t$, which leads to a contradiction.
Corollary 22. Let $\mathscr{B}$ be a quasi-Banach space, $p \in[1, \infty)$, and $t>0$. Then the following conditions are equivalent:

(1) $\mathscr{B}$ is uniformly nonsquare.

(2) $\xi_{\mathscr{B}}^{(p)}(t)<1+t$.

(3) $\xi\left(t, x_{1}\right)<1+t$.

Theorem 23. A quasi-Banach apace $\mathscr{B}$ is uniformly smooth if

$$
\lim _{t \rightarrow 0}\left(\frac{\xi_{\mathscr{B}}^{(p)}(t)-1 / C}{t}\right)=0 .
$$

Proof. Suppose that

$$
\lim _{t \rightarrow 0}\left(\frac{\xi_{\mathscr{B}}^{(p)}(t)-1 / C}{t}\right)=0 .
$$

From Theorem 18, we know that

$$
\xi_{\mathscr{B}}^{(p)}(t) \geq \rho_{\mathscr{B}}(t)+\frac{1}{C},
$$

which implies that

$$
\xi_{\mathscr{B}}^{(p)}(t)-\frac{1}{C} \geq \rho_{\mathscr{B}}(t)
$$

dividing both sides by $t$ and applying the $\lim _{t \rightarrow 0}$

$$
\lim _{t \rightarrow 0} \frac{\rho_{\mathscr{B}}(t)}{t} \leq \lim _{t \rightarrow 0}\left(\frac{\xi_{\mathscr{B}}^{(p)}(t)-1 / C}{t}\right)=0 .
$$

So by definition $\mathscr{B}$ is uniformly smooth.

Theorem 24. Let $\mathscr{B}$ be a quasi-Banach space. Then for $\tau \in$ $(0,1)$

$$
\xi_{\mathscr{B}}^{(p)}(2)<\left[\frac{1}{2} \xi_{\mathscr{B}}^{(p)}(1)+2\right]^{1 / 2}
$$

Proof. If $a, b \in[0,2]$, then $((a+b) / 2)^{2} \geq a^{2}+b^{2}-4$ holds true.

Let $x, y \in S_{\mathscr{B}}$ and $\tau \in[0,1]$. Then we have

$$
\begin{aligned}
& \frac{\|x+\tau y\|}{C} \leq 2, \\
& \frac{\|x-\tau y\|}{C} \leq 2
\end{aligned}
$$

and we have

$$
\begin{aligned}
& {\left[\frac{\|x+\tau y\|+\|x-\tau y\|}{2 C}\right]^{2} \geq\|x+\tau y\|^{2}+\|x-\tau y\|^{2}-4} \\
& \frac{\|x+\tau y\|^{2}+\|x-\tau y\|^{2}}{2 C} \\
& \leq \frac{1}{2}\left(\frac{\|x+\tau y\|+\|x-\tau y\|}{2 C}\right)^{2}+2 .
\end{aligned}
$$


Therefore, we have

$$
\xi_{\mathscr{B}}^{(p)}(2)<\left[\frac{1}{2} \xi_{\mathscr{B}}^{(p)}(1)+2\right]^{1 / 2} .
$$

Theorem 25. Let $\mathscr{B}$ be a quasi-Banach space. Then

$$
\xi_{\mathscr{B}}^{(p)}(1)<\left[\frac{\xi^{p}+2^{p}}{2 C}\right]^{1 / p} .
$$

Proof. Since

$$
\begin{aligned}
& {\left[\frac{\|x+y\|^{p}+\|x-y\|^{p}}{2 C}\right]^{1 / p}} \\
& \quad \leq\left[\frac{\min \left\{\|x+y\|^{p},\|x-y\|^{p}\right\}+2^{t}}{2 C}\right]^{1 / p},
\end{aligned}
$$

therefore, we have

$$
\xi_{\mathscr{B}}^{(p)}(1)<\left[\frac{\xi^{p}+2^{p}}{2 C}\right]^{1 / p} .
$$

\section{Conflicts of Interest}

The authors declare that they have no conflicts of interest.

\section{Authors' Contributions}

All authors contributed equally to the writing of this paper. All authors read and approved the final manuscript.

\section{Acknowledgments}

This work was supported by the Dong-A University research fund.

\section{References}

[1] J. A. Clarkson, "Uniformly convex spaces," Transactions of the American Mathematical Society, vol. 40, no. 3, pp. 396-414, 1936.

[2] L. Zhao and Z. Zhang, "Generalized modulus of smoothness in Banach spaces," Journal of Harbin University of Science and Technology, vol. 21, no. 4, pp. 112-116, 2016.

[3] C. Yang and F. Wang, "On a generalized modulus of convexity and uniform normal structure," Acta Mathematica Scientia B, vol. 27, no. 4, pp. 838-844, 2007.

[4] C. Yang and F. Wang, "On a new geometric constant related to the von Neumann-Jordan constant," Journal of Mathematical Analysis and Applications, vol. 324, no. 1, pp. 555-565, 2006.

[5] C. Yang and F. Wang, "On estimates of the generalized Jordanvon Neumann constant of Banach spaces," Journal of Inequalities in Pure and Applied Mathematics, vol. 7, no. 1, p. 5, 2006.

[6] Z. Zuo and Y. Cui, "A coefficient related to some geometric properties of a Banach space," Journal of Inequalities and Applications, vol. 2009, Article ID 934321, 14 pages, 2009.
[7] Y. C. Kwun, Q. Mehmood, W. Nazeer, A. U. Haq, and S. M. Kang, "Relations between generalized von Neumann-Jordan and JAMes constants for quasi-Banach spaces," Journal of Inequalities and Applications, vol. 171, p. 10, 2016.

[8] W. Nazeer, Q. Mehmood, S. M. Kang, and A. U. Haq, "Generalized von Neumann-Jordan and James constants for QuasiBanach spaces," Journal of Computational Analysis and Applications, vol. 25, no. 6, pp. 1043-1052, 2018.

[9] H. M. U. A. Qadri and Q. Mehmood, "On moduli and constants of quasi-Banach space," Open Journal of Mathematical Science, In Press.

[10] S. M. Kang, H. M. U. A. Qadri, W. Nazeer, and A. U. Haq, "On modulus of convexity of quasi-Banach spaces," Journal of Compututional Analysis and Applications, vol. 25, no. 4, pp. 925934, In Press. 


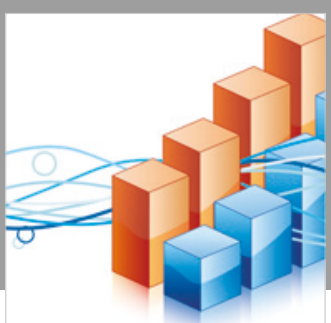

Advances in

Operations Research

\section{-n-m}
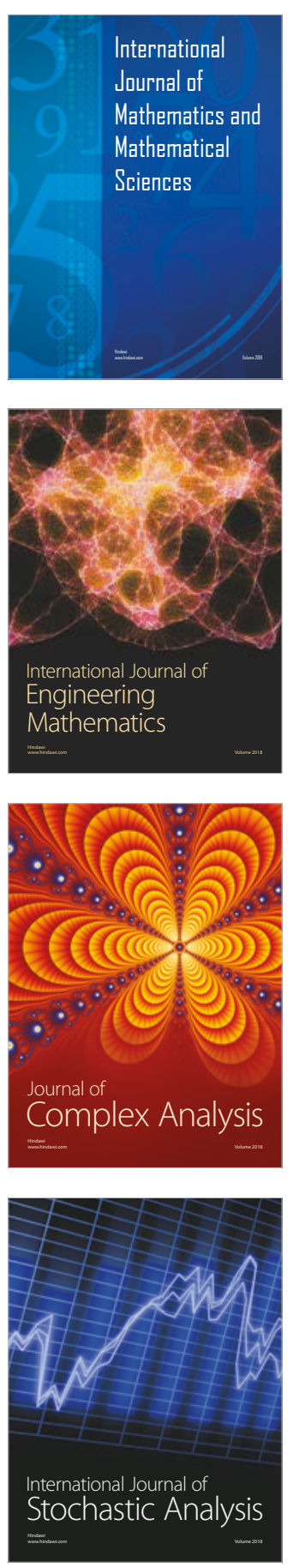
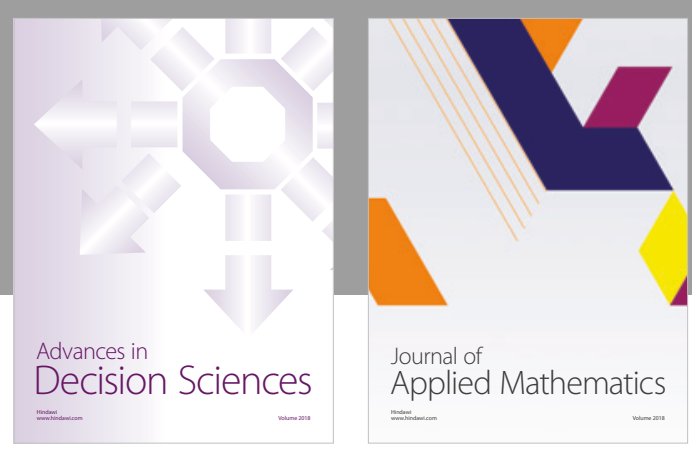

Journal of

Applied Mathematics
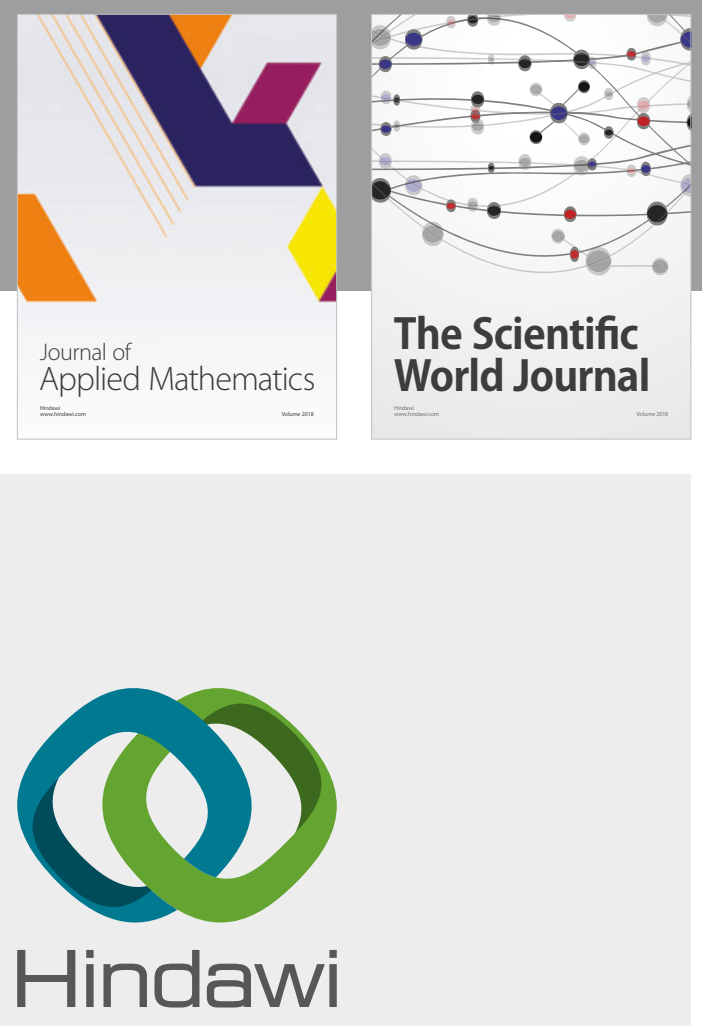

Submit your manuscripts at

www.hindawi.com

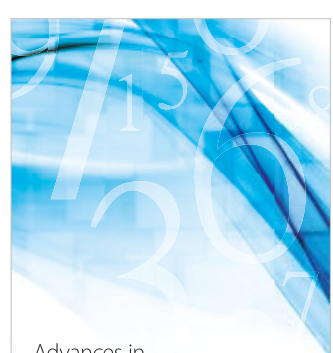

Advances in
Numerical Analysis
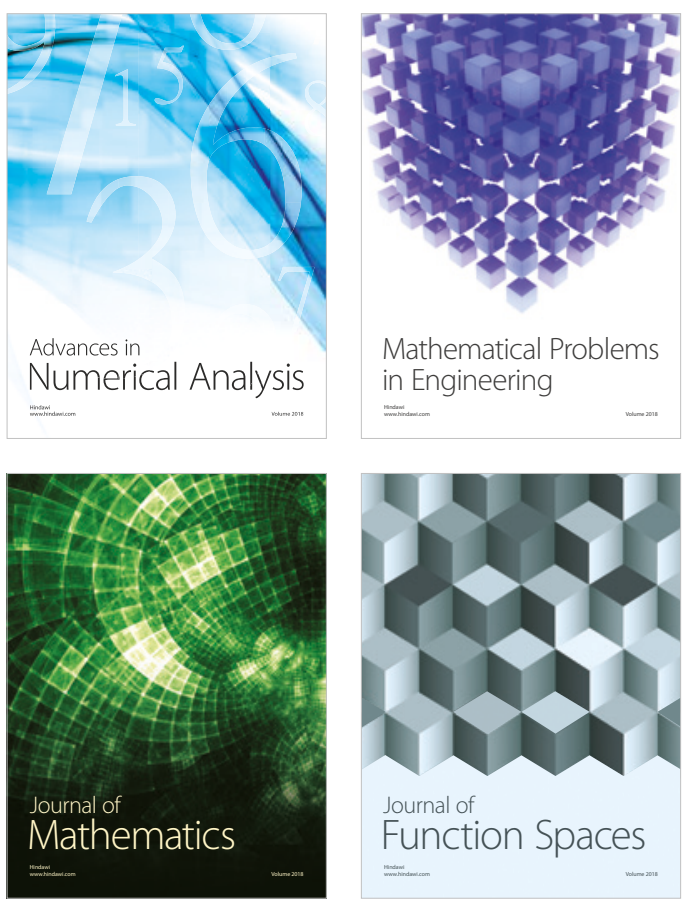

Mathematical Problems in Engineering

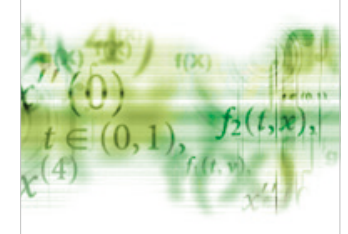

International Journal of

Differential Equations

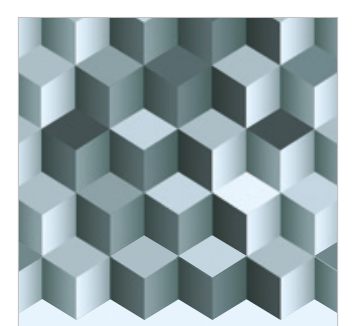

Journal of

Function Spaces

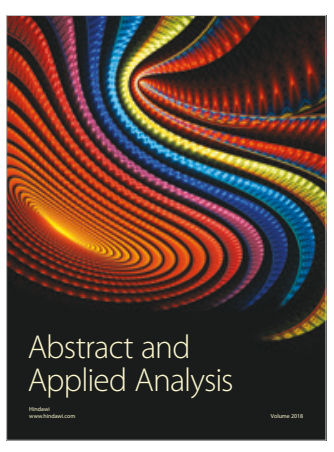

The Scientific

World Journal

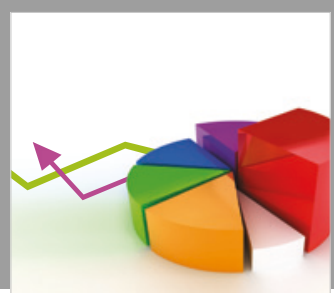

Journal of

Probability and Statistics
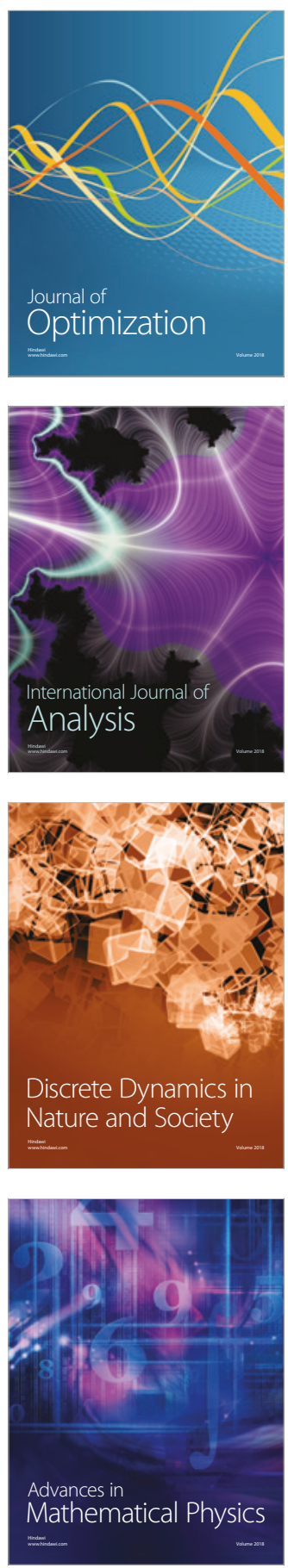